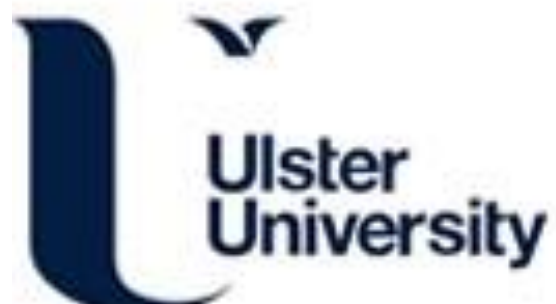

\section{Revealing the dynamic relationship between neural population activities in corticoraphe system}

Behera, C., O'Sullivan, R., Sanchez-Bornot, J., Joshi, A., Prasad, G., Sharp, T., \& Wong-Lin, K. (2020). Revealing the dynamic relationship between neural population activities in corticoraphe system. 1-6. Paper presented at ISSC 2020 - 31st Irish Signals and Systems Conference, Letterkenny, Ireland. https://doi.org/10.1109/ISSC49989.2020.9180170

Link to publication record in Ulster University Research Portal

Publication Status:

Published (in print/issue): 31/08/2020

DOI:

10.1109/ISSC49989.2020.9180170

\section{Document Version}

Author Accepted version

\section{General rights}

Copyright for the publications made accessible via Ulster University's Research Portal is retained by the author(s) and / or other copyright owners and it is a condition of accessing these publications that users recognise and abide by the legal requirements associated with these rights.

\section{Take down policy}

The Research Portal is Ulster University's institutional repository that provides access to Ulster's research outputs. Every effort has been made to ensure that content in the Research Portal does not infringe any person's rights, or applicable UK laws. If you discover content in the Research Portal that you believe breaches copyright or violates any law, please contact pure-support@ulster.ac.uk. 


\title{
Revealing the Dynamic Relationship Between Neural Population Activities in Corticoraphe System
}

\author{
Chandan K. Behera ${ }^{1 *}$, Ruairi O’Sullivan ${ }^{2 *}$, Jose M. Sanchez-Bornot ${ }^{1}$, Alok Joshi ${ }^{1}$, Girijesh Prasad ${ }^{1}$, Trevor Sharp ${ }^{2+}$, \\ and KongFatt Wong-Lin ${ }^{1+}$ \\ Intelligent Systems Research Centre, Ulster University, Magee Campus, Derry Londonderry, Northern Ireland, UK \\ Department of Pharmacology, University of Oxford, Oxford, UK \\ E-mail: \{behera-c,jm.sanchez-bornot, a.joshi, g.prasad, k.wong-lin\}@ulster.ac.uk, ruairi.osullivan@univ.ox.ac.uk, \\ trevor.sharp@pharm.ox.ac.uk \\ *Joint first authors; ${ }^{+}$Corresponding authors
}

\begin{abstract}
Studies have shown that the firing activity of single neurons in brainstem dorsal raphe nucleus (DRN) is linked to slow-wave oscillations in the cortex, especially the frontal cortex. However, most studies consist of either single DRN neuronal or single-channel electrocorticogram (ECoG) recording. Hence, it is unclear how a population of DRN neurons with electrophysiologically diverse characteristics can coordinate and relate to the oscillatory rhythms in different cortical regions. In this work, we explored the technical feasibility of such an investigation. We simultaneously recorded extracellularly a group of DRN neurons and three cortical regions using electrocorticogram (ECoG) in two anaesthetized SERT-Cre mice. The cortical regions were the two bi-hemispheric frontal and one (right) occipital regions. We then used coherence analysis to quantify the relationship between DRN neurons and cortical activity rhythms. We also computed the coherence between firing activities of DRN neurons to quantify their relationship. We found slow-firing DRN neurons with regular and irregular spiking characteristics, potentially serotonergic neurons, were more likely to have stronger relationships with cortical ECoG signals, especially the frontal cortex. Moreover, the DRN neurons were generally found to be weakly correlated with each other. Future investigation with more samples and analytical methods will be conducted to validate our results.
\end{abstract}

Keywords - Dorsal raphe nucleus, neuronal firing activity, cortical oscillations, cortico-subcortical coherence, neuronal firing correlation

\section{INTRODUCTION}

Serotonin (5-HT) is a class of endogenous neurochemicals, called neuromodulators, that can modulate neural activities, which in turn can affect cognition, emotion and behaviour [1][4]. Sources of 5-HT in the brain largely arise from the raphe nuclei deep in the brain, including the dorsal raphe nucleus (DRN) located in the brainstem [1]. It has been shown that there is heterogeneity of DRN neurons in terms of electrophysiological and neurochemical characteristics [5]-[9].

The DRN receives several inputs from various parts of the brain [10], [11] including from the prefrontal cortex (PFC) [12][16]. There are evidences that indicate the prefrontal corticoraphe projection could be mediated by glutamatergic synapses [14], [17]. Further, high frequency stimulation of pyramidal neurons in the PFC is shown to inhibit 5-HT activities in the DRN [12], [18]. More precise state-of-the-art optogenetic stimulation of the PFC has shown potent effects on the DRN activity and behaviour [17], [19], which may have implications in brain disorders, especially the dysfunctions in mood regulation and stress processing [17], [19], [20], as also reflected in abnormal neural activity oscillatory patterns [21].

At the other end of the PFC-DRN circuit, 5-HT-producing neurons from the DRN are known to innervate the cortex, providing dense projection to the frontal cortex [13]. Electrical stimulation of the DRN releases 5-HT that modulates both the frequency and amplitude of cortical slow-wave oscillations in the prefrontal cortex (PFC) [13], [22]-[24]. This slow-wave activity is normally present during natural sleep but can also be induced by certain anaesthetics like urethane [25]. It has also been found that $5-\mathrm{HT}_{1 \mathrm{~A}}$ receptors, a subtype of 5 -HT receptors, mediate decrease in the firing rate of fast spiking interneurons in the PFC, whereas 5- $\mathrm{HT}_{2 \mathrm{~A}}$ receptors mediate increase in the firing rate of fast-spiking inhibitory interneurons in the PFC [26], but overall increase the signal power of cortical slow-wave oscillations [13].

In [27], it reveals that most DRN 5-HT neurons, including those with clock-like and bursting firing activities, are found to have significant coherence with cortical oscillations. Specifically, these neurons typically fire more frequently during the inactive phase (trough) of the slow cortical oscillation. Interestingly, almost $50 \%$ of the bursting 5-HT neurons do not show any significant coherence with cortical rhythms. In contrast, the non-5-HT neurons in the DRN fire at a higher rate during the active phase (peak) of the slow cortical waves. Hence, within the DRN, electrophysiologically and neurochemically distinct neuronal groups exhibit distinct relations to cortical activity.

Overall, the abovementioned evidences seem to indicate a tight reciprocal relationship between the cortex, especially the PFC, and the DRN. However, most of the studies typically involved single-cell recordings and/or focused on slow-wave cortical activity. Moreover, previous studies did not take into account several cortical regions in parallel. Thus, it is not clear how the DRN neuronal population as a whole work in concert with the cortex, and how different cortical regions are comparatively associated with the DRN activity.

To address this, in this work we explore the technical feasibility for such a line of investigation. This is done through 
simultaneous recording of DRN neuronal population's firing activity in conjunction with the monitoring of electrocorticograms (ECoGs) across multiple cortical regions, while also exploring the feasibility of using pairwise coherence analysis to provide insights into the relationships between DRN neuronal firing activities and ECoGs.

\section{METHODS}

\section{A. Experiment}

The open-source Open Ephys tool [28] was used to record the ECoGs in two mice which were urethane-anaesthetised and SERT-Cre (CRE recombinase expressed under control of the serotonin transporter SERT promoter). We used anaesthetized animals because the data was more stable to analyse. SERT-Cre mice were used due to their availability for testing. Simultaneously, extracellular electrophysiological recordings were done using 32 channels using a silicon probe (Cambridge NeuroTech, 32 channels) stereotaxically implanted into the DRN (-4mm posterior to brema). The recordings were done continuously for 1-hour for each session with sampling rate, $\mathrm{Fs}=30 \mathrm{KHz}$.

ECoG electrodes ( 3 channels) were placed bilaterally over the frontal cortex and right occipital cortex to record brain state activities (frontal channels: $+1 \mathrm{~mm}$ anterior and $+-1.5 \mathrm{~mm}$ lateral to bregma; occipital channel: $-2.5 \mathrm{~mm}$ posterior and $+1.5 \mathrm{~mm}$ lateral to bregma). The frontal cortices were selected based on previous studies showing their interactions with the DRN, while the (right) occipital cortex was selected based on previous study showing 5-HT influence in this brain region [29]. Further, the frontal cortex is well-known for high-level top-down cognitive control [30] while the occipital cortex is more for bottom-up sensory (visual) processing [31], very different functional roles.

\section{B. Data Preprocessing}

Raw neuronal spiking data acquired from the 32 channels were filtered and single units were identified automatically using Kilosort [32] and verified by manual clustering using the software package phy [33]. Spike trains were further analysed to reveal spike waveform characteristics, firing rate and firing regularity.

The spiking activities over time, or spike trains, of DRN neurons were labelled and grouped based on their corresponding subtypes, namely, slow regular, slow irregular, fast regular, fast irregular. Instantaneous firing rates (IFR) of the DRN neurons were derived from the corresponding neuronal spike trains using non overlapping time bins of $5 \mathrm{~ms}$, using the Elephant toolbox in Python 3.0 [34].

The 3 ECoG signals were band limited to $25 \mathrm{~Hz}$ using a $5^{\text {th }}$ order Butterworth high-pass filter, because we were interested in low-frequency oscillations and the signals were then concatenated for analysis. No further filtration or average referencing methods were used, which would impart spurious results based on the nature of our dataset (low-density recording, and sensors were not close to each other).
Power spectral analysis of the ECoG signals showed that most of the signal powers were concentrated at the lower frequency components. This was consistent with the nature of our experimental data - the use of anaesthetized mice having brain waves around the delta frequency band [27], [35]. Hence, we focused our analyses on the lower frequencies between 0.5 to $4 \mathrm{~Hz}$.

To assess the relationship between simultaneously recorded neuronal activities between two brain regions (cortex and DRN) we perform coherence analysis [27], [36] (see below). We also computed the coherence between each DRN neurons to find whether the DRN neurons were correlated with each other. We then used standard statistical analysis to find the statistical significance of our measures. These are described in detail below.

\section{Data Analysis}

Coherence analysis, which is performed in frequency space by applying Fourier transform, is a well-known method to compute the frequency dependent relationship (correlation) between two signals [37]. Coherence measures the degree of linear dependency of two signals by testing for similar frequency components.

In our data, the sensors for acquiring the neural activities were neither too many in number nor were they spatially close to each other. Hence, volume conduction may not present a serious issue, and we shall use the magnitude of coherency $(\mathrm{COH})$ to find the interactions between any two neurons. Specifically, the coherence function, $\mathrm{COH}$, at each given frequency $x$ is mathematically described by

$$
C O H(x)=\frac{\left|S_{A B}(x)\right|^{2}}{S_{A A}(x) \cdot S_{B B}(x)}
$$

where $\left|S_{A B}(x)\right|$ is the cross-spectrum between signals A and B, $S_{A A}(x)$ is the autospectrum of signal $\mathrm{A}$, and $S_{B B}(x)$ is the autospectrum of signal B.

\section{Statistical Analysis}

In order to test whether the interaction between the neurons as depicted by the coherence analyses are statistically significant, we calculated the threshold above which coherence level is considered to be statistically significant with $\mathrm{p}<0.01$. To do this, suppose that $T_{1}, T_{2}, \ldots T_{n}$ are test numbers and $P_{1}$, $\mathrm{P}_{2}, \ldots \mathrm{P}_{\mathrm{n}}$ are the corresponding $\mathrm{p}$-values, then the test corresponding to the maximum $\mathrm{p}$-value is calculated as $\mathrm{T}_{\max }$.

While comparing two signals such as IFR and ECoG and to find the coherence estimates inferred from simultaneous trials, we first computed the distribution of $\mathrm{T}_{\max }$. This gives the original statistics of the coherence indices [38]. After that, the $T_{\max }$ for the surrogate data was computed in a similar manner by deriving the surrogate data from the original data. This was done by keeping one signal, e.g. the IFR to be the same as the original while permuting the other signal (e.g. ECoG) randomly. The procedure was repeated for all the three combinations of the ECoG signals and the corresponding $T_{\max }$ values were calculated. The absolute value of these $T_{\max }$ was then found. 
This process was repeated for 1000 Monte Carlo resampling. The $99 \%$ percentile value of these $T_{\max }$ 's was taken as the threshold, which corresponds to p-value equals 0.01 . The tests having $\mathrm{p}<0.01$, were considered to be statistically significant.

\section{RESULTS}

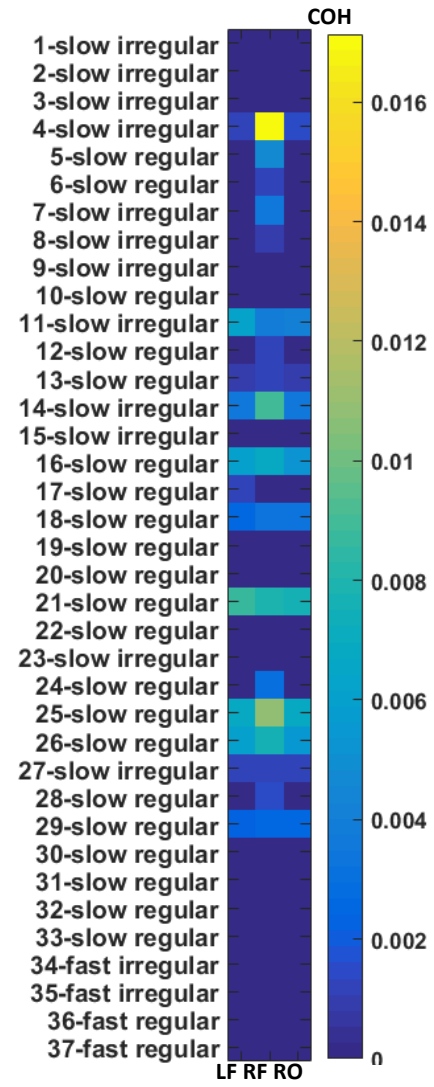

Fig. 1. Interaction between DRN neuronal firing activities and 3 cortical regions. Interactions, measured by the magnitude of coherence $(\mathrm{COH})$, between different subgroups of DRN neurons (vertical axis) and ECoG signals (horizontal axis). Colour bar: $\mathrm{COH}$ level. $\mathrm{LF}$ (RF): $\mathrm{ECoG}$ from left (right) frontal cortices; RO: ECoG from right occipital cortex. DRN neuronal subgroups based on slow regular, slow irregular, fast regular, and fast irregular firing characteristics. Interaction is analysed for frequency range between 0.5 to $4 \mathrm{~Hz}$.

As discussed earlier, the DRN consists of electrophysiologically distinct subgroups of neurons. Specifically, in our recordings, we have identified 4 different subgroups of DRN neurons, namely: (i) fast and irregular spiking; (ii) slow and regular spiking; (iii) slow and irregular spiking; and (iv) fast and regular spiking. We then computed, within the $0.5-4 \mathrm{~Hz}$ frequency band, the coherence of individual neurons with the 3 ECoG signals for each mouse.

Fig. 1 shows the coherence analysis for one mouse in one sample recording session breaking down into the individual neurons labelled by their electrophysiological (spiking) characteristics and the 3 ECoG channels. Coherence magnitudes are plotted against the frequencies to find the frequency at which the signals are more correlated. The channels 43, 44 and 46 were located on the left frontal (LF), right frontal (RF) and right occipital (RO) cortices, respectively. In this session there were 37 neuronal activities. We could easily observe that the slow and regular, and slow and irregular neurons were the majority of neurons in the session. In general, one could observe that the right frontal cortex generally exhibited the highest coherence with the DRN neurons.

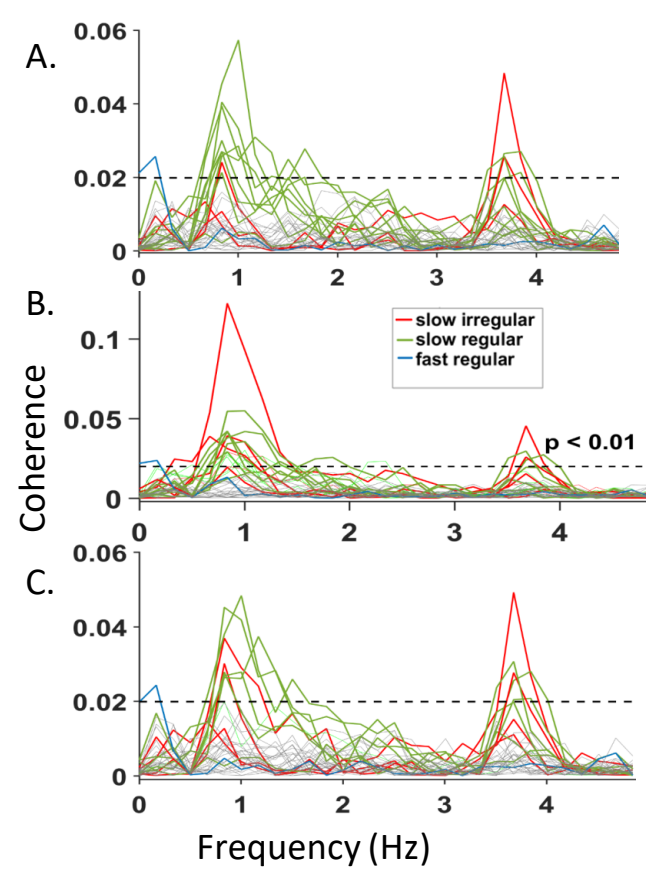

Fig. 2. Double frequency peaks for significant $C O H$ between the DRN neurons and cortical ECoGs. Only slow-regular, slow-irregular, and fast-regular firing neurons are shown. Threshold for significant coherence $(0.02)$ is determined by maximum statistic (see II D). A-C. Interactions between DRN neurons and ECoG signals in LF (A), RF (B) and RO (C). Only statistically significant traces are coloured.

For a more detailed evaluation of the coherences, we plotted in Fig. 2, for the same sample recording session, the coherences across a continuous range of oscillation frequencies, up till $5 \mathrm{~Hz}$. This is shown for all the 37 recorded neurons and their coherences with the left frontal cortex (LF) (Fig. 2A), right frontal corte (RF) (Fig. 2B) and right occipital (RO) cortex (Fig. $2 \mathrm{C})$. We found that the coherences between ECoG activities and DRN neuronal firing rates generally have statistically significant (Fig. 2, above black dashed lines) peaks at around 0.5-1 Hz and 3.5-3.8 Hz, with the former generally higher than the latter. This observation was also consistent with a previous work using extracellular single-cell recording [27]. Interestingly, we found a fast-regular firing DRN neuron to have very weak but significant coherence with the ECoG at a much lower frequency of $0.17 \mathrm{~Hz}$ (Fig. 2, blue). 
For one slow and irregular neuron, the peak at $1 \mathrm{~Hz}$ was substantially higher $(0.12)$ than that at $3.6 \mathrm{~Hz}(0.045)$. In terms of the contribution of the individual DRN neuronal types, other than the fast and irregular spiking DRN neurons, the slow and regular, slow and irregular, and fast and, at a more moderate level, regular spiking types, were found to have relatively significant coherence level with the right frontal ECoG signals (Fig. 1). We found similar patterns, but weaker in coherence magnitudes, for the left frontal and right occipital cortices for the same recording session or mouse (Figs. 2A and 2C).

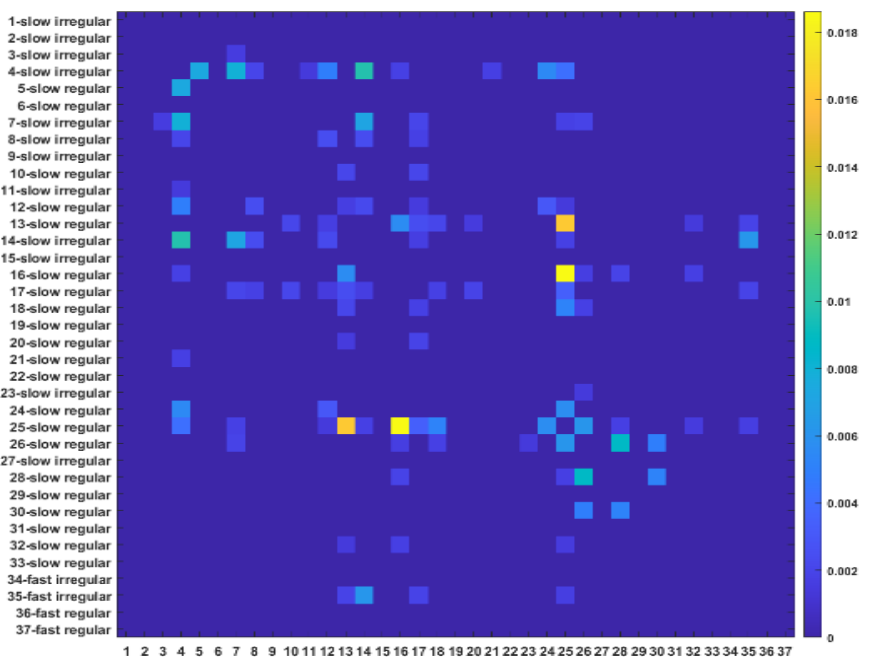

Fig. 3. Sparse and very weak interactions among 37 simultaneously recorded $D R N$ neurons based on magnitude of coherence. Colour bar: $\mathrm{COH}$ level. Most pair of DRN neurons have very low coherence magnitudes (less than 0.018 ), indicating weak interactions. Threshold for significant coherence $(0.02)$ is determined by maximum statistic (see Section II D).

We have shown the variability of coherences between the DRN neuronal subgroups and the ECoG signals, particularly more strongly with the right frontal cortex. To further understand whether the same simultaneously recorded DRN neurons are functionally linked to each other, $\mathrm{COH}$ was computed between the IFRs of every pair of DRN neurons within the same recording session. We found that the coherence matrix was relatively sparse (Fig. 3), with only a few relatively stronger interactions between slow-regular firing DRN neurons (Fig. 3, between orange and yellow). Hence, the DRN neuronal connectivity overall seemed to be potentially sparse, perhaps indicating very weak interactions among the DRN neurons.

By repeating the analytical methods for a different mouse in a different session, similar patterns were observed (Figs. 4-6). As in the results from the previous data, slow-firing DRN neurons have stronger couplings with the right frontal cortical region (Fig. 4). Figs. 5 show that significant interactions with the ECoG signal mainly come from slow-firing DRN neurons. Again, the right frontal cortical ECoG showed the strongest interaction with the DRN neurons, consistent with Fig. 4. However, for this data, the double frequency peaks for the high coherences were not as apparent (compare with Fig. 2), but

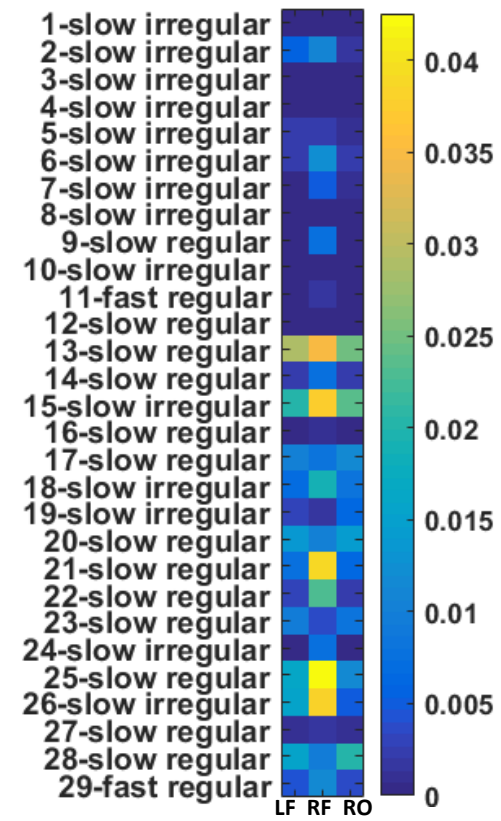

Fig. 4. Interaction between DRN and 3 cortical regions. 29 neurons in this recording session. Labels as in Fig. 1.
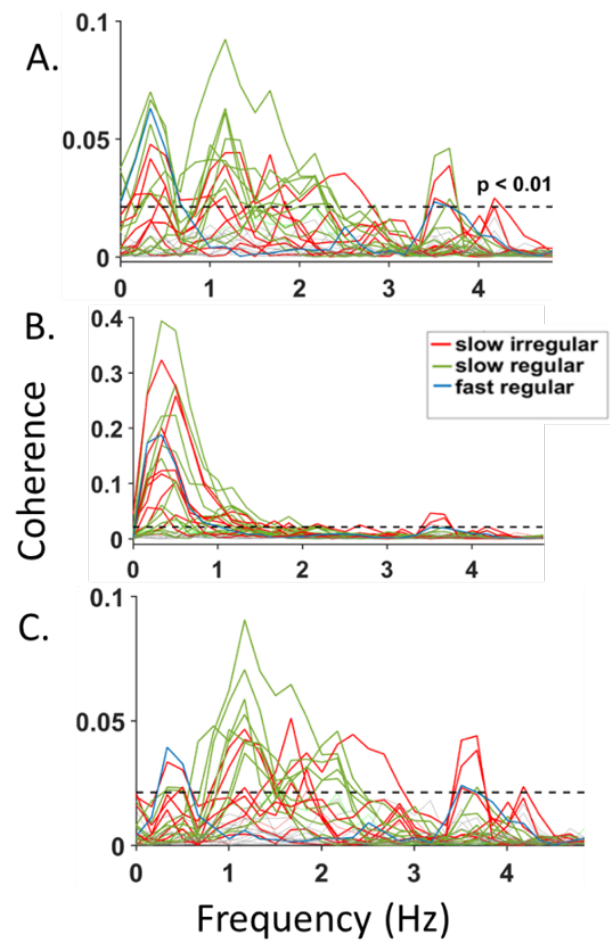

Fig. 5. Majority of significant $\mathrm{COH}$ with ECoGs come from slow firing DRN neurons. Labels as in Fig. 2.

more prominently peaking around $0.5-1.5 \mathrm{~Hz}$. Further, as with our previous data, we observed that the DRN neurons were sparsely and weakly interacting with each other, with their very 
weak coherence magnitudes, and that the neuronal pairs with stronger interactions mainly consisted of slow-regular DRN firing neurons (Fig. 6). A key difference with the previous data was that there were now more slow-regular firing DRN neurons with stronger relationships between each other (Fig. 6), and also with the RF (Fig. 4).

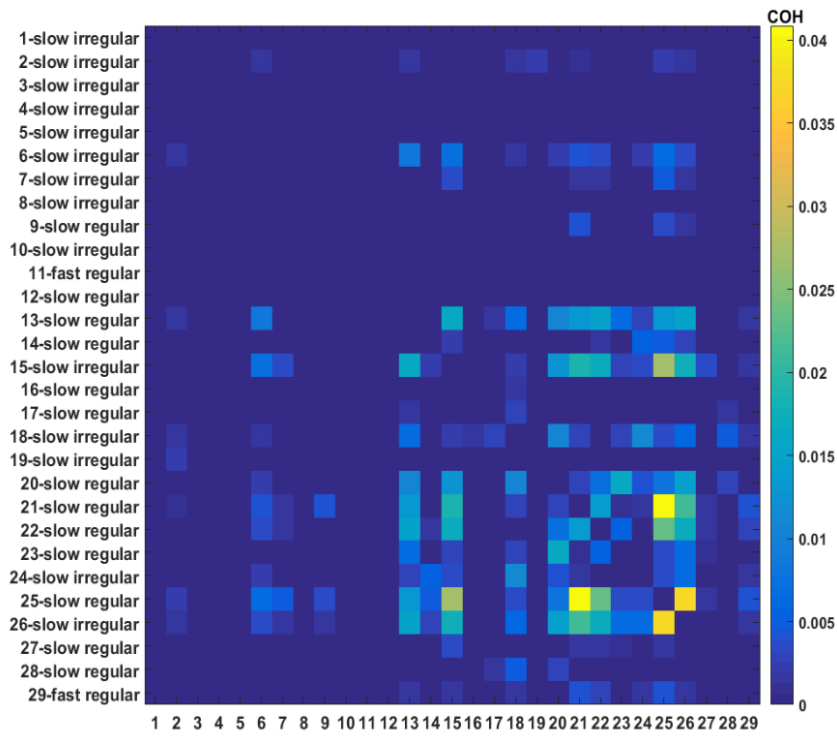

Fig. 6. Sparse and very weak interactions among 29 simultaneously recorded $D R N$ neurons based on magnitude of coherence. Labels as in Fig. 3.

\section{CONCLUSION AND DISCUSSION}

Previous studies have indicated a relationship between the frontal cortex, particularly the PFC, and the DRN neurons [12], [39]. However, most of these studies had investigated using either single-neuron recordings [24] or single ECoG channel [27]. Hence, it is not clear how the diverse DRN neuronal population work together to coordinate, and communicate with cortical rhythms, and the relative contributions of the electrophysiologically distinct DRN neuronal types.

In this work, we successfully tested the technical feasibility of experimentally recording simultaneous DRN neurons and multiple cortical regions (ECoGs) in two anaesthesised mice. We have also successfully applied coherence analytical method to reveal any significant relationship between DRN neurons and between DRN and ECoGs.

Using coherence analysis, we showed that the firing activities of the simultaneously recorded DRN neurons were linked to the slow neural oscillations in the cortex as reflected in the ECoG signals. In particular, we showed that slow (regular and irregular) firing DRN neurons coupled more strongly with ECoG signals, especially the right frontal cortex. Cortical-DRN interactions seemed to operate at a low frequency band of 0.5-1 $\mathrm{Hz}$, which was consistent with our previous work [27]. In addition, our current work also revealed another peak at a slightly higher frequency band of 3.5-3.8 Hz.

5-HT neurons in the DRN typically exhibit slow regular or irregular firing characteristics [6], [40], [41], and so the identified slow-firing DRN neurons could potentially be 5-HT neurons. Future work will confirm this. Given that there were only a small proportion of the DRN neurons in every session found to have significant coherence with the ECoG signals, we checked whether the same group of DRN neurons had low level of communications with each other. Computing using similar coherence analysis, our results showed that only a small proportion of the recorded DRN neurons were found to be correlated with each other. This finding was reminiscent of a recent work which indicated low correlation between pairs of neurons in the locus coeruleus brain region which consisted of another type of monoaminergic neurons, the norepinephrine/noradrenergic neurons [23].

In summary, our work has demonstrated technical feasibility, both experimentally and analytical, to understand the dynamic relationship between neurons and neuronal populations in the corticoraphe system. Future work would entail more recording sessions and mice, including wild-type mice, and more minority neuronal subgroups (e.g. fast irregular spiking). Also, given that the animals were anaesthetised in this study, future work should seek to apply our methods to identify the dynamic interactions between the DRN and the cortex in awake or behaving animals.

\section{ACKNOWLEDGMENT}

This work was supported by IEEE Computational Intelligence Society Graduate Student Research Grant, and BBSRC (BB/P003427/1). CKB was supported by Ulster University Research Challenge Fund.

\section{REFERENCES}

[1] B. L. J. Müller, Christian P., Handbook Of Behavioral Neuroscience, vol. 21, no. ISBN: 978-0-12-374634-4. 2010.

[2] P. Dayan and Q. J. M. Huys, "Serotonin in Affective Control," Annu. Rev. Neurosci., vol. 32, no. 1, pp. 95-126, 2009.

[3] P. De Deurwaerdère and G. Di Giovanni, "Serotonergic modulation of the activity of mesencephalic dopaminergic systems: Therapeutic implications," Prog. Neurobiol., vol. 151, pp. 175-236, 2017.

[4] K. Wong-Lin, D.-H. Wang, A. A. Moustafa, J. Y. Cohen, and K. Nakamura, "Toward a multiscale modeling framework for understanding serotonergic function," J. Psychopharmacol., p. 026988111769961, 2017.

[5] B. W. Okaty, K. G. Commons, and S. M. Dymecki, "Embracing diversity in the 5-HT neuronal system.," Nat. Rev. Neurosci., vol. 20, no. 7, pp. 397-424, Jul. 2019.

[6] K. A. Allers and T. Sharp, "Neurochemical and anatomical identification of fast- and slow-firing neurones in the rat dorsal raphe nucleus using juxtacellular labelling methods in vivo," Neuroscience, vol. 122, no. 1, pp. 193-204, Nov. 2003.

[7] B. Kocsis, V. Varga, L. Dahan, and A. Sik, "Serotonergic neuron diversity: Identification of raphe neurons with discharges time-locked to the hippocampal theta rhythm," Proc. Natl. Acad. Sci. U. S. A., vol. 103, no. 4, pp. 1059-1064, 2006.

[8] Y.-Q. Li, H. Li, T. Kaneko, and N. Mizuno, "Morphological features and 
electrophysiological properties of serotonergic and non-serotonergic projection neurons in the dorsal raphe nucleus: An intracellular recording and labeling study in rat brain slices," Brain Res., vol. 900, no. 1, pp. 110118, 2001.

[9] S. Marinelli, "Serotonergic and Nonserotonergic Dorsal Raphe Neurons Are Pharmacologically and Electrophysiologically Heterogeneous," $J$. Neurophysiol., vol. 92, no. 6, pp. 3532-3537, 2004.

[10] I. Pollak Dorocic et al., "A Whole-Brain Atlas of Inputs to Serotonergic Neurons of the Dorsal andMedian Raphe Nuclei," Neuron, vol. 83, no. 3, pp. 663-678, 2014

[11] S. P. Ranade and Z. F. Mainen, "Transient firing of dorsal raphe neurons encodes diverse and specific sensory, motor, and reward events.," $J$. Neurophysiol., vol. 102, no. 5, pp. 3026-3037, Nov. 2009.

[12] P. Celada, M. V Puig, J. M. Casanovas, G. Guillazo, and F. Artigas, "Control of dorsal raphe serotonergic neurons by the medial prefrontal cortex: Involvement of serotonin-1A, GABA(A), and glutamate receptors," J Neurosci, vol. 21, no. 24, pp. 9917-9929, 2001.

[13] P. Celada, M. V. Puig, and F. Artigas, "Serotonin modulation of cortical neurons and networks," Front. Integr. Neurosci., vol. 7, p. 25, Apr. 2013.

[14] C. Challis and O. Berton, "Top-Down Control of Serotonin Systems by the Prefrontal Cortex: A Path toward Restored Socioemotional Function in Depression.," ACS Chem. Neurosci., vol. 6, no. 7, pp. 1040-1054, Jul. 2015.

[15] M. Hajós, C. D. Richards, A. D. Székely, and T. Sharp, "An electrophysiological and neuroanatomical study of the medial prefrontal cortical projection to the midbrain raphe nuclei in the rat," Neuroscience, vol. 87, no. 1, pp. 95-108, 1998.

[16] C. A. Heidbreder and H. J. Groenewegen, "The medial prefrontal cortex in the rat: evidence for a dorso-ventral distinction based upon functional and anatomical characteristics.," Neurosci. Biobehav. Rev., vol. 27, no. 6, pp. 555-579, Oct. 2003.

[17] S. D. Geddes et al., "Target-specific modulation of the descending prefrontal cortex inputs to the dorsal raphe nucleus by cannabinoids," Proc. Natl. Acad. Sci. U. S. A., vol. 113, no. 19, pp. 5429-5434, 2016.

[18] J. C. Shaw, "An introduction to the coherence function and its use in EEG signal analysis," J. Med. Eng. Technol., vol. 5, no. 6, pp. 279-288, Jan. 1981.

[19] M. R. Warden et al., "A prefrontal cortex-brainstem neuronal projection that controls response to behavioural challenge.," Nature, vol. 492, no. 7429, pp. 428-432, Dec. 2012.

[20] L. R. Srejic, K. M. Wood, A. Zeqja, P. Hashemi, and W. D. Hutchison, "Modulation of serotonin dynamics in the dorsal raphe nucleus via high frequency medial prefrontal cortex stimulation," Neurobiol. Dis., vol. 94, pp. 129-138, 2016

[21] E. Basar and B. Guntekin, "A review of brain oscillations in cognitive disorders and the role of neurotransmitters.," Brain Res., vol. 1235, pp. 172-193, Oct. 2008.

[22] P. Celada, M. V. Puig, L. Diaz-Mataix, and F. Artigas, "The hallucinogen DOI reduces low-frequency oscillations in rat prefrontal cortex: reversal by antipsychotic drugs.," Biol. Psychiatry, vol. 64, no. 5, pp. 392-400, Sep. 2008.

[23] N. K. Totah, R. M. Neves, S. Panzeri, N. K. Logothetis, and O. Eschenko, "The Locus Coeruleus Is a Complex and Differentiated Neuromodulatory System.," Neuron, vol. 99, no. 5, pp. 1055-1068.e6, Sep. 2018.

[24] S. E. Gartside, E. Hajos-Korcsok, E. Bagdy, L. G. J. Harsing, T. Sharp, and M. Hajos, "Neurochemical and electrophysiological studies on the functional significance of burst firing in serotonergic neurons.," Neuroscience, vol. 98, no. 2, pp. 295-300, 2000.

[25] J. Crook and T. Lovick, "Urodynamic function during sleep-like brain states in urethane anesthetized rats.," Neuroscience, vol. 313, pp. 73-82, Jan. 2016.

[26] M. V. Puig, A. Watakabe, M. Ushimaru, T. Yamamori, and Y. Kawaguchi, "Serotonin Modulates Fast-Spiking Interneuron and Synchronous Activity in the Rat Prefrontal Cortex through 5-HT 1A and 5-HT_2A Receptors," J. Neurosci., vol. 30, no. 6, pp. 2211 LP-2222, Feb. 2010 .

[27] J. V. Schweimer, N. Mallet, T. Sharp, and M. A. Ungless, "Spike-timing relationship of neurochemically-identified dorsal raphe neurons during cortical slow oscillations," Neuroscience, vol. 196, pp. 115-123, 2011.

[28] J. H. Siegle, A. C. Lopez, Y. A. Patel, K. Abramov, S. Ohayon, and J. Voigts, "Open Ephys: an open-source, plugin-based platform for multichannel electrophysiology.," J. Neural Eng., vol. 14, no. 4, p. 45003, Aug. 2017.

[29] G. Jonsson, A. Gorio, H. Hallman, D. Janigro, H. Kojima, and R. Zanoni, "Effect of GM1 ganglioside on neonatally neurotoxin induced degeneration of serotonin neurons in the rat brain," Dev. Brain Res., vol. 16, no. 2, pp. 171-180, 1984.

[30] S. Funahashi, "Working Memory in the Prefrontal Cortex," Brain Sci., vol. 7, no. 5, p. 49, Apr. 2017.

[31] M. A. Nazari et al., "Visual sensory processing deficit in the occipital region in children with attention-deficit/hyperactivity disorder as revealed by event-related potentials during cued continuous performance test," Neurophysiol. Clin. Neurophysiol., vol. 40, no. 3, pp. 137-149, 2010.

[32] M. Pachitariu, N. Steinmetz, S. Kadir, M. Carandini, and H. Kenneth D., "Kilosort: realtime spike-sorting for extracellular electrophysiology with hundreds of channels," bioRxiv, p. 61481, Jan. 2016

[33] C. Rossant et al., "Spike sorting for large, dense electrode arrays," Nat. Neurosci., vol. 19, no. 4, pp. 634-641, 2016.

[34] A. Yegenoglu, M. Denker, S. Grun, L. D. Phan, A. Davison, and D. Holstein, "Elephant - Open-Source Tool for the Analysis of Electrophysiological Data Sets," in INM Retreat 2015, 2015, p. 26.

[35] M. Steriade, "Corticothalamic resonance, states of vigilance and mentation," Neuroscience, vol. 101, no. 2, pp. 243-276, 2000.

[36] S. M. Bowyer, "Coherence a measure of the brain networks: past and present," Neuropsychiatr. Electrophysiol., vol. 2, no. 1, pp. 1-12, 2016.

[37] J. R. Rosenberg, A. M. Amjad, P. Breeze, D. R. Brillinger, and D. M. Halliday, "The Fourier approach to the identification of functional coupling between neuronal spike trains.," Prog. Biophys. Mol. Biol., vol. 53, no. 1, pp. 1-31, 1989.

[38] T. Nichols and S. Hayasaka, "Controlling the familywise error rate in functional neuroimaging: a comparative review.," Stat. Methods Med. Res., vol. 12, no. 5, pp. 419-446, Oct. 2003.

[39] M. V Puig, F. Artigas, and P. Celada, "Modulation of the activity of pyramidal neurons in rat prefrontal cortex by raphe stimulation in vivo: Involvement of serotonin and GABA," Cereb. Cortex, vol. 15, no. 1, pp. $1-14,2005$.

[40] B. Mlinar, A. Montalbano, L. Piszczek, C. Gross, and R. Corradetti, "Firing Properties of Genetically Identified Dorsal Raphe Serotonergic Neurons in Brain Slices.," Front. Cell. Neurosci., vol. 10, p. 195, 2016.

[41] M. Hajós et al., "Neurochemical identification of stereotypic burst-firing neurons in the rat dorsal raphe nucleus using juxtacellular labelling methods," Eur. J. Neurosci., vol. 25, no. 1, pp. 119-126, 2007. 